\title{
Effects of PLK1 on proliferation, invasion and metastasis of gastric cancer cells through epithelial-mesenchymal transition
}

\author{
RUI SONG, GE HOU, JUN YANG, JINJIN YUAN, CHENG WANG, TING CHAI and ZONGWEN LIU \\ Department of Tumor Radiotherapy, The Second Affiliated Hospital of Zhengzhou University, \\ Zhengzhou, Henan 450014, P.R. China
}

Received January 12, 2018; Accepted July 31, 2018

DOI: $10.3892 / 01.2018 .9406$

\begin{abstract}
Effects of polo-like kinase (PLK1) on proliferation, migration and invasion capacities of gastric cancer cells through epithelial-mesenchymal transition (EMT) were investigated. Small-interfering ribonucleic acid (siRNA) with targeted interference in PLK1 gene was designed and transfected into gastric cancer MGC-803 cells via Lipofectamine to inhibit the expression of PLK1 gene in MGC-803 cells. The proliferation of MGC-803 cells was detected via methyl thiazolyl tetrazolium (MTT) assay. The mRNA and protein expression of PLK1 and EMT-related marker (E-cadherin) was detected via real-time polymerase chain reaction and western blot analysis, respectively. The effects of interference in PLK1 gene on migration and invasion of MGC-803 cells were studied via wound healing assay and Transwell chamber assay, respectively. Results of MTT assay showed that compared with that in control group, the cell proliferation in PLK1 siRNA group was significantly inhibited $(\mathrm{p}<0.01)$. Compared with those in control group, the mRNA and protein expression of PLK1 in PLK1 siRNA group was significantly decreased $(\mathrm{p}<0.01)$, but the mRNA and protein expression of E-cadherin was obviously upregulated $(\mathrm{p}<0.01)$. Results of wound healing assay and invasion assay showed that the capacity of migration and invasion of MGC-803 cells in PLK1 siRNA group was significantly inhibited compared with those in control group $(\mathrm{p}<0.01)$. In conclusion, PLK1 enhances the proliferation, migration and invasion of gastric cancer MGC-803 cells through affecting EMT.
\end{abstract}

Correspondence to: Dr Zongwen Liu, Department of Tumor Radiotherapy, The Second Affiliated Hospital of Zhengzhou University, 2 Jingba Road, Jinshui, Zhengzhou, Henan 450014, P.R. China

E-mail: liuzongwen06@163.com

Key words: PLK1, gastric cancer cells, epithelial-mesenchymal transition, proliferation and invasion

\section{Introduction}

It was found in epidemiological statistics that the mortality rate of gastric cancer in malignant tumors is only lower than that of lung cancer, and its incidence rate ranks fourth in the world $(1,2)$. The clinical treatment means of early gastric cancer is mainly surgery, but most patients are in the advanced stage when diagnosed, so the chemotherapy-based comprehensive treatment is mainly adopted. Unfortunately, chemotherapy does not significantly improve the survival rate of patients with advanced gastric cancer, and patients have to face the adverse effects brought by chemotherapy (3-5). Gastric cancer does not have typical clinical features in the early stage, so it is often neglected. There are reports showing that $50-60 \%$ of patients with gastric cancer in China are in the advanced stage, and the resection rate is only $40 \%$ in diagnosed patients. Due to a high malignant degree of tumor, gastric cancer is still vulnerable to recurrence and metastasis even after resection, there is a poor prognosis, and only $20-30 \%$ of patients can live for 5 years after surgery. Therefore, the effect of simple surgical treatment is often disappointing for patients with advanced gastric cancer, especially for those who have undergone local tumor spread or metastasis to other tissues (6). Currently, the clinical treatment of gastric cancer has been developed from simple surgical treatment to comprehensive treatment of surgery and chemoradiotherapy, and individualized and targeted therapies have become hot spots in treatment (7).

The process in which epithelial cells differentiate into cells with biological characteristics of mesenchymal phenotype according to a certain procedure is called epithelial-mesenchymal transition (EMT). EMT is a key link in invasion and distant spread of tumor cells (8). Polo-like kinase (PLK) is a kind of serine/threonine protein kinase, which widely exists in eukaryotic cells with certain cycle dependence. Its name is derived from the fact that it can lead to abnormality in the formation of spindle body in Drosophila (9). Numerous studies have shown that PLK1 plays an important role in chromosome segregation, promotion of centrosome maturation, cytokinesis and other processes. However, the roles of PLK1 in the occurrence and development of cancer have not been elucidated.

The experiments were carried out to investigate the effects of PLK1 on the proliferation, migration and invasion of gastric 
cancer cells, and to explore the roles of EMT in the proliferation, migration and invasion processes of gastric cancer cells.

\section{Materials and methods}

Materials and reagents. Human gastric cancer cell lines MGC-803 (Cell Bank of Chinese Academy of Sciences, Shanghai, China), methyl thiazolyl tetrazolium (MTT) (Sigma, St. Louis, MO, USA), glyceraldehyde-3-phosphate dehydrogenase (GAPDH), PLK1 and E-cadherin human primary antibodies and horseradish peroxidase (HRP)-labeled second antibodies (all from Wuhan Sanying Biotechnology, Wuhan, China), primer synthesis (Takara Biotechnology Co., Ltd., Dalian, China), Dulbecco's modified Eagle's medium (DMEM) (Gibco Life Technologies, Carlsbad, CA, USA), ribonucleic acid (RNA) extraction kits, reverse transcription kits and reverse transcription-polymerase chain reaction (RT-PCR) kits (all from from Invitrogen; Thermo Fisher Scientific, Inc., Carlsbad, CA, USA), bicinchoninic acid (BCA) protein quantification kits and cell lysis solution (all from Beyotime Institute of Biotechnology, Nantong, China). The study was approved by the Ethics Committee of The Second Affiliated Hospital of Zhengzhou University (Zhengzhou, China) and written informed consents were signed by the patients and/or guardians.

Design and construction of PLK1 expression vectors. PLK1 and negative control plasmids (siNC) were designed and synthesized by Shanghai GenePharma Co., Ltd., (Shanghai, China). The sense strand of PLK1 small-interfering ribonucleic acid (siRNA) is 5'-GCAACCUGCAGUGUAAUAATT-3', and its antisense strand is 5'-UUAUUACACUGCAGGUUG CTT-3'. The sense strand of siNC is 5'-GCCTCAACATCC CCTACAAGA-3', and its antisense strand is: 5'-CCACGA AGAACAGAAGCACAAA-3'.

Cell culture. MGC-803 cells were cultured routinely using DMEM containing $10 \%$ fetal bovine serum (FBS) in an incubator with $5 \% \mathrm{CO}_{2}$ at $37^{\circ} \mathrm{C}$. When cells were in the logarithmic growth phase, they were digested by trypsin, prepared into single-cell suspension and inoculated into a culture dish at an appropriate density. Cells in this experiment were divided into 3 groups: Blank control (no transfection), negative control (transfected with siNC) and experimental group (transfected with siPLK1).

Detection of cell proliferation inhibition rate. After transfection, cells were inoculated into a 96-well plate at a density of $1 \times 10^{5} / \mathrm{ml}(100 \mu \mathrm{l}$ per well). After $48 \mathrm{~h}, 10 \mu \mathrm{l}$ MTT $(5 \mathrm{mg} / \mathrm{ml})$ was added into each well and cells were cultured for another $4 \mathrm{~h}$. The optical density (OD) value of each well at a wavelength of $570 \mathrm{~nm}$ was detected using a microplate reader (Bio-Rad Laboratories, Inc., Hercules, CA, USA). The cell proliferation inhibition rate was calculated as follows: Inhibition rate $(\%)=\left(\mathrm{OD}\right.$ value blank control group OD value experi- $_{\text {- }}$ mental group $/ \mathrm{OD}$ value $\mathrm{blank}_{\text {control group }}$ ) $100 \%$.

Detection of PLK1 and E-cadherin MRNA expression via $R T$-qPCR. After transfection, cells were inoculated into a 6 -well plate $\left(10^{4} /\right.$ well $)$, the supernatant was discarded after
Table I. RT-PCR primer sequences.

\begin{tabular}{ll}
\hline Genes & \multicolumn{1}{c}{ Primer sequence } \\
\hline E-cadherin & F: 5'-GCTTGGAATGAGACTGCTGA-3' \\
& R: 5'-CTGGCCATATCCACCAGAGT-3' \\
PLK1 & F: 5'-CGAGGGTGATGAGAACCTGC-3' \\
& R: 5'-CCCATGTGATTCGATGCGT-3' \\
GAPDH & F: 5'-CAAGGTCATCCATGACAACTTTG-3' \\
& R: 5'-GTCCACCACCCTGTTGCTGTAG-3'
\end{tabular}

F, forward; R, reverse.

$48 \mathrm{~h}$, and cells in each group were collected. The total RNA was extracted from tissues according to the instructions of RNA extraction kit, and the concentration and purity of total RNA were determined using an ultraviolet-visible spectrophotometer (Hitachi, Ltd., Tokyo, Japan) (qualified if $\mathrm{A}_{260} / \mathrm{A}_{280}>1.8$ ). The complementary deoxyribonucleic acid (cDNA) was obtained via reverse transcription according to the instructions of reverse transcription kit, and then the cDNA was used as a template to detect the mRNA expression of PLK1 and E-cadherin according to the instructions of RT-PCR kit. The primer sequences are shown in Table I, and the reaction conditions are as follows: $95^{\circ} \mathrm{C}$ for $10 \mathrm{~min}, 95^{\circ} \mathrm{C}$ for $15 \mathrm{sec}, 60^{\circ} \mathrm{C}$ for $1 \mathrm{~min}$, and amplification for 40 cycles. The cycle threshold $(\mathrm{Cq})$ value was the output from the instrument software, and the relative expression level was calculated using $2^{-\Delta \Delta \mathrm{Cq}}$ method according to the following formula: $\Delta \Delta \mathrm{Cq}$ (target gene) $=\mathrm{Cq}$ (target gene) $-\mathrm{Cq}$ (control gene) (10)

Detection of protein expression of PLKI and E-cadherin via western blot analysis. After transfection, cells were inoculated into the 6 -well plate $\left(10^{4} /\right.$ well), and the supernatant was discarded after $48 \mathrm{~h}$. Then cells in each group were collected, lysed with the cell lysis solution, and centrifuged at $8,000 \mathrm{xg}$ at $4^{\circ} \mathrm{C}$ for $15 \mathrm{~min}$, and the supernatant was collected. The protein concentration was determined using the BCA kit, followed by degeneration, separation via sodium dodecyl sulfate polyacrylamide gel electrophoresis (SDS-PAGE), and membrane transfer using the wet method. Then the membrane was sealed via bull serum albumin (BSA) for $2 \mathrm{~h}$, and Rabbit anti-human GAPDH, PLK1 and E-cadherin polyclonal antibodies (cat. nos. 10494-1-AP, 10305-1-AP and 20874-1-AP; 1:1,000; Wuhan Sanying Biotechnology) was added for incubation at $4^{\circ} \mathrm{C}$ overnight. After the membrane was washed, goat anti-rabbit polyclonal secondary antibody (cat. no. SA00001-2; 1:2,000; Wuhan Sanying Biotechnology) was added for incubation at room temperature for $2 \mathrm{~h}$. After the membrane was washed again, the image was developed in a darkroom via enhanced chemiluminescence (ECL), scanned and recorded using a gel imager (Bio-Rad Laboratories, Richmond, CA, USA). The gray scale was analyzed and compared with GADPH as internal reference.

Detection of migration capacity of human gastric cancer MGC-803 cells via wound healing assay. After transfection, cells were inoculated into the 6 -well plate $\left(10^{4} /\right.$ well). After 
Table II. Inhibitory effect of transfection with PLK1 siRNA for $48 \mathrm{~h}$ on MGC-803 cell proliferation (mean $\pm \mathrm{SD}$ ).

\begin{tabular}{lccc}
\hline & \multicolumn{3}{c}{ Proliferation inhibition rate $(\%)$} \\
\cline { 2 - 4 } Groups & $24 \mathrm{~h}$ & $48 \mathrm{~h}$ & $72 \mathrm{~h}$ \\
\hline Blank control & 0 & 0 & 0 \\
Negative control & 0 & 0 & 0 \\
PLK1 siRNA & $11.3 \pm 0.32^{\mathrm{a}}$ & $23.32 \pm 2.13^{\mathrm{a}}$ & $37.33 \pm 2.06^{\mathrm{a}}$ \\
\hline Compared with control & group, ${ }^{\mathrm{a}} \mathrm{p}<0.01$. & PLK1, polo-like kinase; \\
siRNA, small-interfering ribonucleic acid.
\end{tabular}

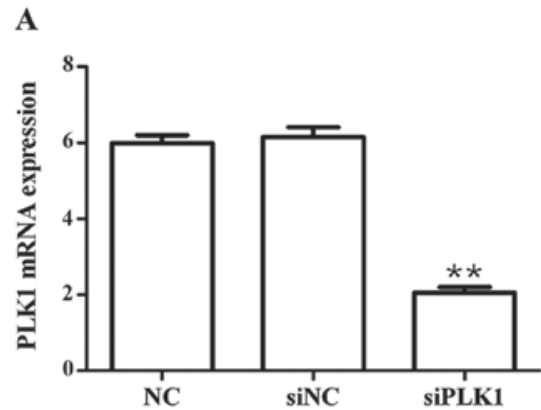

B

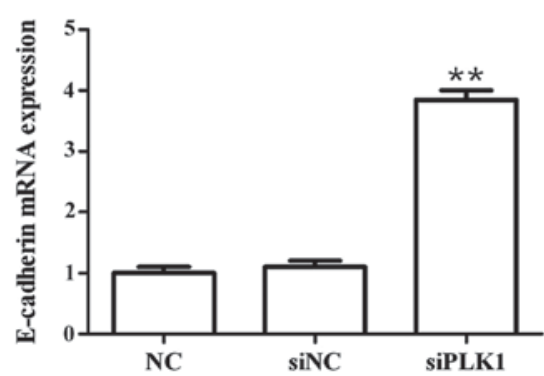

Figure 1. Detection of effects of siPLK1 transfection on mRNA expression of PLK1 and E-cadherin via RT-PCR. (A) PLK1 mRNA expression level. (B) E-cadherin mRNA expression level. Compared with those in blank control group, the PLK1 mRNA expression level is significantly inhibited, but the E-cadherin mRNA expression level is significantly upregulated ${ }^{* *} \mathrm{p}<0.01$. PLK1, polo-like kinase.

about $80 \%$ cells were fused, a $100 \mu 1$ spearhead was used to scratch at the marker line. The loose cells were gently rinsed off with phosphate buffered saline (PBS), and the remaining cells continued to be cultured using FBS-free DMEM culture solution. After $48 \mathrm{~h}$, the distance between scratches were observed and photographed.

Detection of invasion capacity of human gastric cancer MGC-803 cells via Transwell assay. The upper Transwell chamber was evenly added with $100 \mu$ l single-cell suspension in a concentration of $4 \times 10^{5} / \mathrm{ml}$ and $100 \mu \mathrm{l}$ serum-free culture solution, while the lower chamber was added with $500 \mu 1$ FBS-free culture solution. Cells were treated according to the above experimental method. After $48 \mathrm{~h}$, cells were fixed with $4 \%$ paraformaldehyde, stained via crystal violet for $15 \mathrm{~min}$, photographed and analyzed.
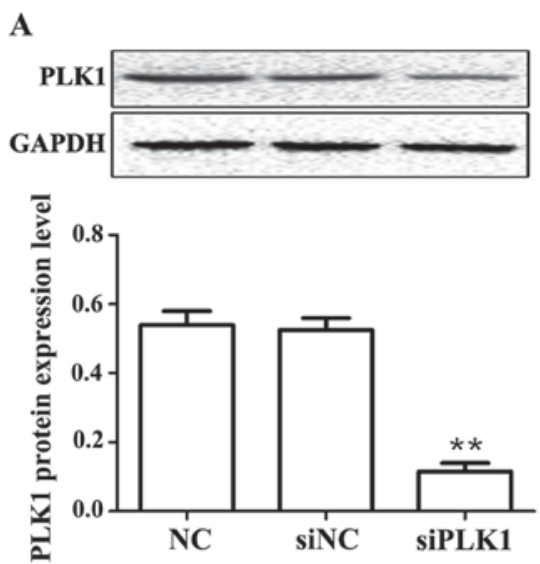

B
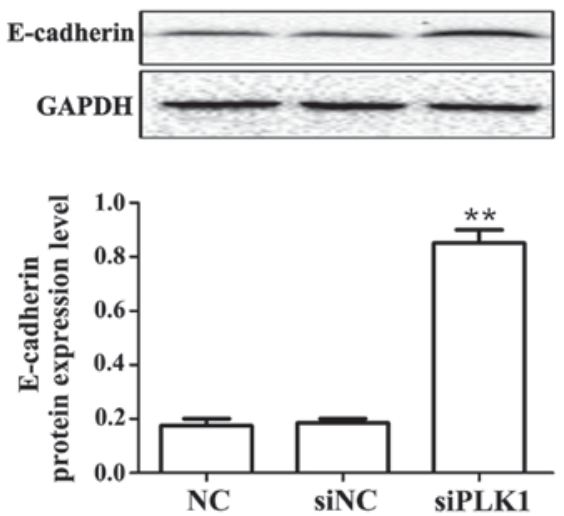

Figure 2. Detection of effects of siPLK1 transfection on protein expression of PLK1 and E-cadherin via western blot analysis. (A) PLK1 protein expression level. (B) E-cadherin protein expression level. Compared with those in blank control group, the PLK1 protein expression level is significantly inhibited, but the E-cadherin protein expression level is significantly upregulated. ${ }^{* *} \mathrm{p}<0.01$. PLK1, polo-like kinase.

Statistical analysis. Data were presented as mean \pm standard deviation (SD) and Statistical Product and Service Solutions (SPSS) 17.0 software (SPSS, Inc., Chicago, IL, USA) was used for data processing. One-way analysis of variance was used for the statistical analysis of data obtained and the post hoc test was Dunnett's test. $\mathrm{P}<0.05$ was considered to indicate a statistically significant difference.

\section{Results}

Effect of siPLK1 on MGC-803 cell proliferation. Results of MTT assay showed that at $48 \mathrm{~h}$ after transfection into MGC-803 cells, the cell inhibition rate in PLK1 siRNA group was significantly increased compared with that in blank control group, and the difference was statistically significant $(\mathrm{p}<0.01)$ (Table II).

Effects of siPLK1 transfection on mRNA expression of PLK1 and E-cadherin. Results showed that compared with those in blank control group, PLK1 siRNA could significantly decrease the mRNA expression of PLK1 $(\mathrm{p}<0.01)$, but significantly upregulate the mRNA expression of E-cadherin $(\mathrm{p}<0.01)$ at $48 \mathrm{~h}$ after transfection into MGC- 803 cells, and the differences were statistically significant (Fig. 1). 


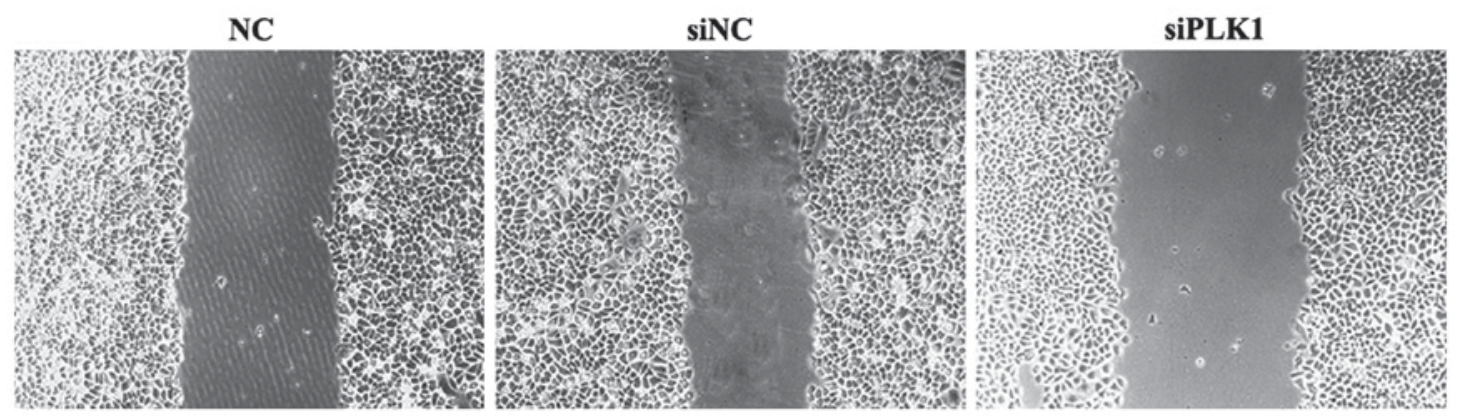

Figure 3. Detection of effect of siPLK1 on migration capacity of MGC-803 cells via wound healing assay. PLK1, polo-like kinase.
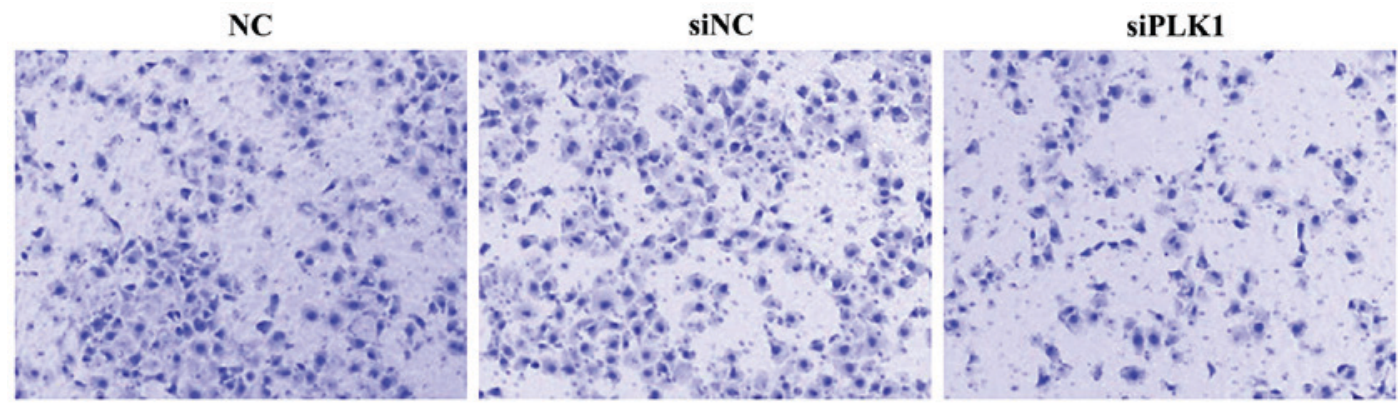

Figure 4. Detection of effect of siPLK1 transfection on cell invasion capacity via Transwell assay. PLK1, polo-like kinase.

Effects of siPLK1 transfection on protein expression of PLKI and E-cadherin. Results showed that compared with those in control group, PLK1 siRNA could obviously decrease the protein expression of PLK1 $(\mathrm{p}<0.01)$, but obviously upregulate the protein expression of E-cadherin $(\mathrm{p}<0.01)$ at $48 \mathrm{~h}$ after transfection into MGC-803 cells, and the differences were statistically significant (Fig. 2).

Effect of siPLK1 transfection on migration capacity of MGC-803 cells. Results of wound healing assay showed that compared with those in blank control and negative control groups, the space between wounds in experimental group was wider, the healing was obviously inhibited, and the cell migration capacitywas weakened. The difference in the wound space was not significant between blank control and negative control groups (Fig. 3).

Effect of siPLK1 transfection on invasion capacityofMGC-803 cells. At $48 \mathrm{~h}$ after transfection into MGC-803 cells, cells were inoculated into the upper Transwell chamber for culture for $48 \mathrm{~h}$. The number of MGC-803 cells passing through Matrigel and reaching the filter membrane in PLK1 siRNA group was significantly smaller than those in blank control and negative control groups, indicating that PLK1 siRNA can inhibit the invasion of MGC- 803 cells. The difference was not significant between blank control and negative control groups (Fig. 4).

\section{Discussion}

Gastric cancer is a common malignant tumor of digestive tract, and its incidence rate is increasing year by year, seriously affecting human health (11). At present, clinical treatment means have not only side effects, but also slow progression, and the mechanisms of the occurrence and development of gastric cancer are not clear. Therefore, exploring new mechanisms is of great significance to find a new breakthrough for gastric cancer treatment.

EMT is a key link in the metastasis of tumor cells, involving the activation of multiple factors and different signaling pathways (12). The adhesion between epithelial cells with EMT is significantly decreased, but the cell motility is significantly enhanced, thus cells further migrate. During the occurrence of EMT in cells, relevant markers are also changed, such as the deletion of epithelial phenotype marker E-cadherin. It has been reported that the typical sign of EMT is the deletion of E-cadherin expression, and this process involves another important factor, namely the zinc-finger transcriptional factor Snail that plays a key role in the EMT process. It is found that Snail can promote the mesenchymal transition of a variety of tumor cells, and the invasion and metastasis of tumor cells $(13,14)$. Zinc-finger transcriptional factor directly binds to the promoter of E-cadherin, thus leading to the downregulation of E-cadherin protein expression, further reducing the adhesion between cells and destroying the physiological tissue structure, thereby promoting the invasion and metastasis of tumor cells (15). In addition, PLK1, as a kinase, is a key factor in mitosis, which is closely associated with multiple processes of mitosis, such as formation of spindle body, centrosome replication and chromosome segregation (16). A large number of research reports (17-19) have shown that the PLK1 expression is increased in various solid tumor tissues (20), such as esophageal cancer, melanoma, breast cancer, colon cancer and renal cell carcinoma, suggesting that there is a close relationship between PLK1 and tumor occurrence. Smith et al transfected the exogenously-recombinant PLKl genes into normal fibroblasts, and results showed that malignant transformation of 
cells could be induced, and the tumor could be implanted into nude mice successfully, indicating that the $P L K 1$ gene can directly cause malignant transformation of cells (21). In addition, recent reports have indicated that the high expression of $P L K 1$ gene in liver and pancreatic cancers occurs mainly in the early stage of tumor cell progression (22).

In this experiment, siRNA with targeted interference in PLK1 gene was designed and transfected into gastric cancer MGC-803 cells via Lipofectamine (Thermo Fisher Scientific, Inc., Waltham, MA, USA) to inhibit the expression of PLK1 gene in MGC-803 cells. Results of MTT assay showed that compared with that in control group, the cell proliferation in PLK1 siRNA group was significantly inhibited. Results of real-time PCR and western blot analysis showed that compared with those in control group, the mRNA and protein expression of PLK1 in PLK1 siRNA group were significantly decreased, but the mRNA and protein expression of E-cadherin was obviously upregulated. Results of wound healing assay and invasion assay showed that the migration and invasion of MGC-803 cells in PLK1 siRNA group were significantly inhibited compared with those in control group. It is reported (23) that epithelial cancerous cells will have stronger migration and invasion capacities after EMT. In addition, it has been reported (24) that the upregulation of PLK1 expression will lead to the accumulation of oncogenes in tumor cells and formation of tumors with migration and invasion capacities in nude mice successfully. Clinically, Tokumitsu et al (25) studied the PLK1 mRNA expression level in patients with gastric cancer, and results showed that PLK1 mRNA was highly expressed in $73 \%$ of patients, and the difference was significant compared with that in normal and atypical hyperplastic tissues. Besides, the expression level of PLK1 was positively correlated with the clinical staging and depth of tumor infiltration. All of these reports indicate that PLK1 plays an extremely important role in the occurrence and development of human tumors. Therefore, exploring the mechanism of action of PLK1 in tumors will provide a new direction for further treatment of tumors.

In conclusion, this study proved that PLK1 can enhance the proliferation, migration and invasion of gastric cancer MGC-803 cells through affecting EMT.

\section{Acknowledgements}

Not applicable.

\section{Funding}

No funding was received.

\section{Availability of data and materials}

All data generated or analyzed during this study are included in this published article.

\section{Authors' contributions}

RS and GH designed the study, JUY and JIY collected and analysed the data, CW helped with Transwell assay. TC and ZL were responsible for PCR and western blot analysis. All authors read and approved the final manuscript.

\section{Ethics approval and consent to participate}

The study was approved by the Ethics Committee of The Second Affiliated Hospital of Zhengzhou University (Zhengzhou,China) and written informed consents were signed by the patients and/or guardians.

\section{Patient consent for publication}

Not applicable.

\section{Competing interests}

The authors declare that they have no competing interests.

\section{References}

1. Brenner H, Rothenbacher D and Arndt V: Epidemiology of stomach cancer. Methods Mol Biol 472: 467-477, 2009.

2. Jemal A, Siegel R, Ward E, Hao Y, Xu J and Thun MJ: Cancer statistics, 2009. CA Cancer J Clin 59: 225-249, 2009.

3. Ryu MH and Kang YK: ML17032 trial: Capecitabine/cisplatin versus 5-fluorouracil/cisplatin as first-line therapy in advanced gastric cancer. Expert Rev Anticancer Ther 9: 1745-1751, 2009.

4. De Vita F, Vecchione L, Galizia G, Di Martino N, Fabozzi T, Catalano G, Ciardiello F and Orditura M: Perspectives in adjuvant therapy of gastric cancer. Oncology 77 (Suppl 1): 38-42, 2009

5. Mlkvý P: Multimodal therapy of gastric cancer. Dig Dis 28: 615-618, 2010.

6. Mello BS, Lucena AF, Echer IC and Luzia MF: Patients with gastric cancer submitted to gastrectomy: An integrative review. Rev Gaúcha Enferm 31: 803-811, 2010 (In Portuguese).

7. Bang YJ, Van Cutsem E, Feyereislova A, Chung HC, Shen L, Sawaki A, Lordick F, Ohtsu A, Omuro Y, Satoh T, et al; ToGA Trial Investigators: Trastuzumab in combination with chemotherapy versus chemotherapy alone for treatment of HER2-positive advanced gastric or gastro-oesophageal junction cancer (ToGA): A phase 3, open-label, randomised controlled trial. Lancet 376: 687-697, 2010.

8. Xu J, Lamouille S and Derynck R: TGF-beta-induced epithelial to mesenchymal transition. Cell Res 19: 156-172, 2009.

9. de Cárcer G,Escobar B, Higuero AM, García L, Ansón A, Pérez G Mollejo M, Manning G, Meléndez B, Abad-Rodríguez J, et al: Plk5, a polo box domain-only protein with specific roles in neuron differentiation and glioblastoma suppression. Mol Cell Biol 31: 1225-1239, 2011.

10. Livak and Schmittgen: Analysis of relative gene expression data using real-time quantitative PCR and the $2-\Delta \Delta \mathrm{Cq}$ method. Methods 25: 402-408, 2001.

11. Crew KD and Neugut AI: Epidemiology of upper gastrointestinal malignancies. Semin Oncol 31: 450-464, 2004.

12. Spiegel S and Milstien S: Functions of the multifaceted family of sphingosine kinases and some close relatives. J Biol Chem 282: 2125-2129, 2007.

13. Zeisberg M and Neilson EG: Biomarkers for epithelial-mesenchymal transitions. J Clin Invest 119: 1429-1437, 2009.

14. Peinado H, Olmeda D and Cano A: Snail, Zeb and bHLH factors in tumour progression: An alliance against the epithelial phenotype? Nat Rev Cancer 7: 415-428, 2007.

15. Cano A, Pérez-Moreno MA, Rodrigo I, Locascio A, Blanco MJ, del Barrio MG, Portillo F and Nieto MA: The transcription factor snail controls epithelial-mesenchymal transitions by repressing E-cadherin expression. Nat Cell Biol 2: 76-83, 2000.

16. Ando K, Ozaki T, Yamamoto H, Furuya K, Hosoda M, Hayashi S, Fukuzawa $\mathrm{M}$ and Nakagawara A: Polo-like kinase 1 (Plk1) inhibits p53 function by physical interaction and phosphorylation. J Biol Chem 279: 25549-25561, 2004.

17. Steinhauser I, Langer K, Strebhardt K and Spänkuch B: Uptake of plasmid-loaded nanoparticles in breast cancer cells and effect on Plk1 expression. J Drug Target 17: 627-637, 2009.

18. Kim SA, Kwon SM, Yoon JH and Ahn SG: The antitumor effect of PLK1 and HSF1 double knockdown on human oral carcinoma cells. Int J Oncol 36: 867-872, 2010.

19. Takai N, Hamanaka R, Yoshimatsu J and Miyakawa I: Polo-like kinases (Plks) and cancer. Oncogene 24: 287-291, 2005. 
20. Zhang G, Zhang Z and Liu Z: Polo-like kinase 1 is overexpressed in renal cancer and participates in the proliferation and invasion of renal cancer cells. Tumour Biol 34: 1887-1894, 2013.

21. Smith MR, Wilson ML, Hamanaka R, Chase D, Kung H, Longo DL and Ferris DK: Malignant transformation of mammalian cells initiated by constitutive expression of the polo-like kinase. Biochem Biophys Res Commun 234: 397-405, 1997.

22. Petrelli A, Perra A, Schernhuber K, Cargnelutti M, Salvi A, Migliore C, Ghiso E, Benetti A, Barlati S, LeddaColumbano GM, et al: Sequential analysis of multistage hepatocarcinogenesis reveals that miR-100 and PLK1 dysregulation is an early event maintained along tumor progression. Oncogene 31: 4517-4526, 2012.

23. Voulgari A and Pintzas A: Epithelial-mesenchymal transition in cancer metastasis: Mechanisms, markers and strategies to overcome drug resistance in the clinic. Biochim Biophys Acta 1796: 75-90, 2009 .
24. Smits VA, Klompmaker R, Arnaud L, Rijksen G, Nigg EA and Medema RH: Polo-like kinase-1 is a target of the DNA damage check point. Nat Cell Biol 2: 672-676, 2000.

25. Tokumitsu Y, Mori M, Tanaka S, Akazawa K, Nakano S and Niho Y: Prognostic significance of polo-like kinase expression in esophageal carcinoma. Int J Oncol 15: 687-692, 1999.

This work is licensed under a Creative Commons Attribution-NonCommercial-NoDerivatives 4.0 International (CC BY-NC-ND 4.0) License. 